and P2 amplify a fragment of 291 base pairs from the wild-type allele; primers P1 and $\mathrm{P} 3$ amplify a product of $440 \mathrm{bp}$, indicating the presence of the targeted allele. Histological and in situ hybridization studies. Embryos and trophoblast tissues from heterozygous intercrosses were fixed in $4 \%$ paraformaldehyde, dehydrated and embedded in paraffin. Sections $(7 \mu \mathrm{m})$ were stained with haematoxylin and $\operatorname{eosin}^{19}$ or processed for in situ hybridization with radiolabelled riboprobes ${ }^{20}$. Genotypes of embryos were identified by PCR using DNA from embryonic tissues scraped from histological sections.

BrdU labelling of trophoblast tissues. BrdU $(100 \mu \mathrm{g}$ per gram of body weight) was injected intraperitoneally into pregnant female mice. The mice were killed $1 \mathrm{~h}$ later, and the embryos and trophoblast tissues were removed and fixed in $4 \%$ paraformaldehyde and processed for immunohistochemistry. The genotype of each embryo was identified by PCR using DNA from embryonic tissues scraped from sections. Sections were incubated with an anti-BrdU monoclonal antibody ${ }^{21}$ (BROMO-2), and antibody binding was visualized with anti-mouse immunoglobulin-alkaline phosphatase (Boehringer Mannheim). Sections were stained with haematoxylin to visualize nuclei and allow cells to be counted.

Generation of tetraploid aggregation chimaeras. Electrofusion of two-cellstage blastomeres collected from CD1 female mice or CD1 females crossed with male homozygous for the ROSA-26 gene trap insertion ${ }^{22}$ was used to produce wild-type tetraploid embryos. The fused embryos were cultured overnight in embryo culture medium in $5 \% \mathrm{CO}_{2}$ at $37^{\circ} \mathrm{C}$. Eight-cell-stage diploid embryos were recovered from the oviduct of embryonic-day (e) 2.5 pregnant ERR- $\beta$ heterozygote females mated to an ERR- $\beta$ heterozygote male. Two four-cell tetraploid embryos were sandwiched around a diploid eight-cell-stage embryo from the heterozygous cross and aggregated overnight. Successfully aggregated embryos were transferred to e2.5-pseudopregnant CD1 recipients. Recipients were killed at e12.5 and embryos were genotyped and examined. Those aggregates made with ROSA-26 tetraploid embryos were also stained for $\beta$-galactosidase activity in the placenta, yolk sac and embryonic components $^{23}$.

Received 21 March; accepted 15 May 1997.

1. Giguère, V., Yang, N., Segui, P. \& Evans, R. M. Identification of a new class of steroid hormon receptors. Nature 331, 91-94 (1988).

2. Pettersson, K. et al. Expression of a novel member of estrogen response element-binding nuclear receptors is restricted to the early stages of chorion formation during mouse embryogenesis. Mech. Dev. 54, 211-223 (1996).

3. Guillemot, F., Nagy, A., Auerbach, A., Rossant, J. \& Joyner, A. L. Essential role of Mash-2 in extraembryonic development. Nature 371, 333-336 (1994)

4. Lescesin, K. R., Vamuza, S. \& Rossant, J. Isolation and characterization of a novel trophoblast-specific cDNA in the mouse. Genes Dev. 2, 1639-1646 (1988).

Finnerty, H. et al. Molecular cloning of murine FLT and FLT4. Oncogene 8, 2293-2298 (1996).

6. Colosi, P., Swiergiel, J. J., Wilder, E. L., Oviedo, A. \& Linzer, D. I. Characterization of proliferin-related protein. Mol. Endocrinol. 2, 579-586 (1988)

Linzer, D. I., Lee, S. J., Ogren, L., Talamantes, F. \& Nathans, D. Identification of proliferin mRNA and protein in mouse placenta. Proc. Natl Acad. Sci. USA 82, 4356-4359 (1985).

8. Nagy, A., Rosant, J., Nagy, R., Abramow-Newerly, W. \& Roder, J. C. Derivation of completely cell culture-derived mice from early-passage embryonic stem cells. Proc. Natl Acad. Sci. USA 90. 84248428 (1993).

. Nagy, A. et al. Embryonic stem cells alone are able to support fetal development in the mouse. Development 110. 815-821 (1990).

10. Ilgren, E. B. On the control of the trophoblastic giant-cell transformation in the mouse: homotypic cellular interactions and polyploidy. J. Embryol. Exp. Morphol. 62, 183-202 (1981).

11. Rossant, J. \& Ofer, L. Properties of extra-embryonic ectoderm isolated from postimplantation mouse embryos. J. Embryol. Exp. Morphol. 39, 183-194 (1977).

12. Rossant, J. \& Tamura-Lis, W. Effect of culture conditions on diploid to giant-cell transformation in postimplantation mouse trophoblast. J. Embryol. Exp. Morphol. 62, 217-227 (1981).

13. Ilgren, E. B. Control of trophoblast growth. Placenta 4, 307-328 (1983).

14. Yen, S. S. C. \& Jaffe, R. B. Reproductive Endocrinology (Saunders, Philadelphia, 1991).

15. Speroff, L., Glass, R. H. \& Kase, N. G. Clinical Gynecologic Endocrinology and Infertility (Williams \& Wilkins, Baltimore, 1994)

16. Lubahn, D. B. et al. Alteration of reproductive function but not prenatal sexual development after disruption of the mouse estrogen receptor. Proc. Natl Acad. Sci. USA 90, 11162-11166 (1993).

17. Lydon, J. P. et al. Mice lacking progesterone receptor exhibit pleiotropic reproductive abnormalities. Genes Dev. 9, 2266-2278 (1995)

18. Nagy, A. \& Rossant, J. in Gene targeting: a practical approach (ed. Joyner, A. L.) 147-169 (Oxford Univ Press, 1993).

19. Kaufman, M. H. The Atlas of Mouse Development (Academic, London, 1992).

20. Wilkinson, D. G. In situ Hybridization: a Practical Approach (IRL, Oxford, 1992).

21. Paramithiotis, E. \& Ratcliffe, M. J. B cell emigration directly from the cortex of lymphoid follicles in the bursa of Fabricus. Eur. J. Immunol. 24, 458-463 (1994).

22. Friedrich, G. \& Soriano, P. Promoter traps in embryonic stem cells: a genetic screen to identify and mutate developmental genes in mice. Genes Dev. 5, 1513-1523 (1991).

23. Gossler, A., Joyner, A. L., Rossant, J. \& Skarnes, W. C. Mouse embryonic stem cells and reporte constructs to detect developmentally regulated genes. Science 244, 463-465 (1989).

Acknowledgements. We thank M. Tremblay for injection of ES cells; M. Ratcliffe for the anti-BrdU antibody; and K. Kemball for technical advice. J.L. was supported by a fellowship from the MRC of
Canada, and R.S. was supported by an NCIC Terry Fox clinician-scientist fellowship. This work was supported by grants from the MRC of Canada (C.G., J.R.). J.R. is an international scholar of the Howard Hughes Medical Institute and a Terry Fox research scientist of the NCIC. V.G. is an MRC scientist.

Correspondence and requests for materials should be addressed to V.G. (e-mail: vgiguere@dir.molonc. mcgill.ca)

\section{Inflammatory stimuli induce accumulation of MHC class II complexes on dendritic cells}

\author{
Marina Cella, Anneke Engering, Valerie Pinet* \\ Jean Pieters \& Antonio Lanzavecchia \\ Basel Institute for Immunology, Grenzacherstrasse 487, CH 4005 Basel, \\ Switzerland \\ * Laboratoire d'Immunologie, Inserm U-291, Hôpital Saint Eloi, 34295 \\ Montpellier, France
}

Dendritic cells have the remarkable property of presenting any incoming antigen ${ }^{1}$. To do so they must not only capture antigens with high efficiency and broad specificity, but must also maximize their capacity to load class II molecules of the major histocompatibility complex (MHC) with antigenic peptides in order to present a large array of epitopes from different proteins, each at a sufficient copy number. Here we show that formation of peptide-MHC class II complexes is boosted by inflammatory stimuli that induce maturation of dendritic cells. In immature dendritic cells, class II molecules are rapidly internalized and recycled, turning over with a half-life of about 10 hours. Inflammatory stimuli induce a rapid and transient boost of class II synthesis, while the half-life of class II molecules increases to over 100 hours. These coordinated changes result in the rapid accumulation of a large number of long-lived peptide-loaded MHC class II molecules capable of stimulating $T$ cells even after several days. The capacity of dendritic cells to load many antigenic peptides over a short period of initial exposure to inflammatory stimuli could favour presentation of infectious antigens.

A key feature of dendritic cells is that they can capture antigens in peripheral tissues and migrate to secondary lymphoid organs where they acquire the ability to stimulate naive $\mathrm{T}$ cells ${ }^{1}$. This maturation process was originally noted in freshly isolated Langerhans cells which, upon culture in vitro, lost acidic organelles and the capacity to synthesize class II molecules and to present soluble antigen, while acquiring the ability to stimulate $\mathrm{T}$ cells ${ }^{1-6}$. Although the final results of the maturation process is loss of antigen-capturing and -processing capacity, it is not known whether the generation of peptideMHC class II complexes is enhanced during the transition from immature to mature dendritic cells.

We previously described an in vitro culture system suitable for analysing the maturation process of dendritic cells. Human monocytes cultured in medium supplemented with granulocyte-macrophage colony-stimulating factor (GM-CSF) and interleukin (IL)-4 develop into a homogeneous population with the characteristics of immature dendritic cells, namely high endocytic activity and a low capacity for stimulating $\mathrm{T}$ cells. When challenged by inflammatory stimuli such as tumour-necrosis factor (TNF)- $\alpha$, IL-1 and lipopolysaccharide (LPS), these cells can no longer endocytose, they upregulate adhesion and co-stimulatory molecules, and become mature stimulatory dendritic cells ${ }^{7,8}$. We used this system to study the effect of dendritic cell maturation on class II-restricted antigen presentation.

Cell-surface and total class II molecules were quantified in intact and permeabilized cells. As shown in Fig. 1a, immature dendritic cells express high levels of total class II molecules, but only a small fraction is present on the cell surface. During the first 24 hours after 
stimulation with LPS or TNF- $\alpha$, up to $\sim 6 \times 10^{6}$ class II molecules are deposited on the cell surface. This large increase is paralleled by an increase in total class II molecules, suggesting that both redistribution and new synthesis have occurred (Fig. 1b, c).

To investigate the large intracellular class II pool present in immature dendritic cells, we first measured class II internalization and recycling. In immature dendritic cells, biotinylated Fab fragments of an anti-DR antibody bound to surface class II molecules are rapidly internalized and recycled back to the cell surface (Fig. 2a). Within $5 \mathrm{~min}$, about $50 \%$ of the Fab disappears from the cell surface, indicating that the internalization process is very rapid and that the recycling pool is as large as the surface pool. After stimulation with LPS or TNF- $\alpha$, the large pool of recycling class II molecules progressively decreased and disappeared after 40 hours (Fig. 2b). This decrease was paralleled by a decrease in both fluid phase and receptor-mediated endocytic activity (Fig. 2c).

It has been previously shown that different antigenic peptides can be loaded either on newly synthesized ${ }^{9}$ or on recycling class II molecules ${ }^{10-12}$. Given that in immature dendritic cells the pool of recycling class II molecules far exceeds that detected in other antigen-presenting cells (APC) such as B cells ${ }^{10,13,14}$, we determined the relative contribution of recycling and newly synthesized class II molecules in antigen presentation. We pulsed immature dendritic cells at $37^{\circ} \mathrm{C}$ with a low concentration of tetanus toxoid (TT) and
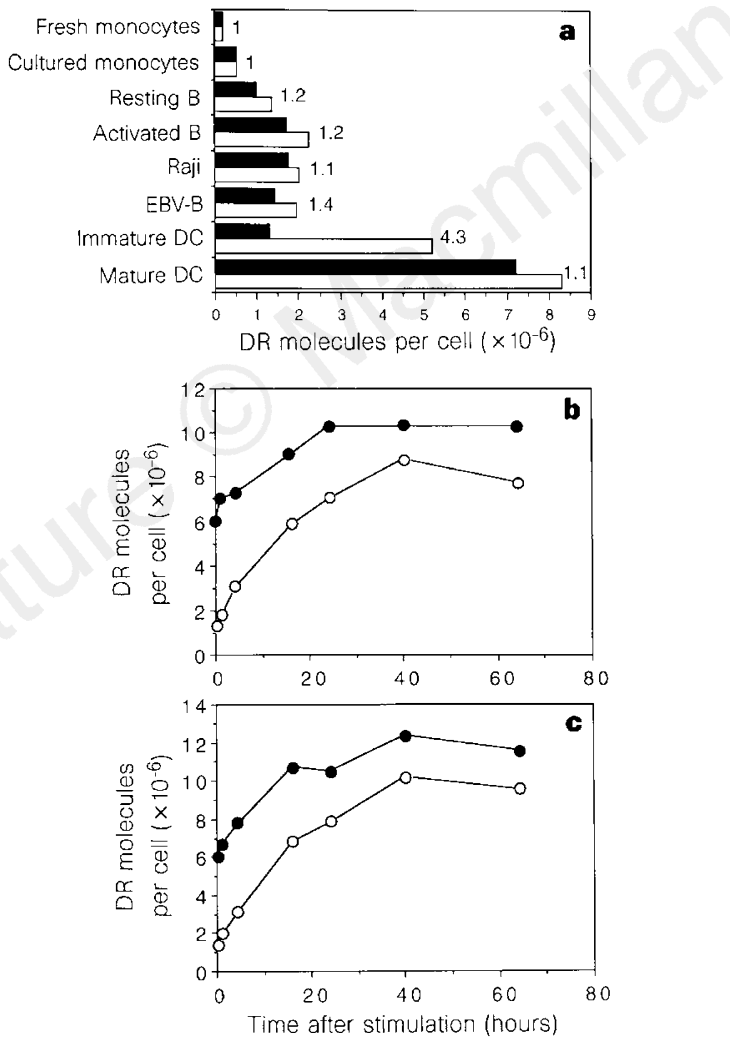

Figure 1 Increased expression of surface and total class II molecules in DC stimulated with LPS or TNF- $\alpha$. a, Number of surface (black bars) and total (white bars) DR molecules in different APC. The number indicates to the total/surface ratio. b, c, Kinetics of increase in surface $(O)$ and total $(\bullet)$ DR molecules in DC stimulated with LPS (b) or TNF- $\alpha$ (c). Results are representative of at least 5 different experiments. measured the formation of specific peptide-class II complexes by following, as an early readout of $\mathrm{T}$ cell activation, the induction of $\mathrm{Ca}^{2+}$ flux in TT-specific T-cell clones ${ }^{15}$. As shown in Fig. 3, the epitope recognized by clone ALT220 was generated very rapidly (20 min after addition of TT), whereas the epitope recognized by clone ALT81 was generated only after 40-50 min. Blocking of protein synthesis by pretreating dendritic cells with cycloheximide did not affect presentation to clone ALT220, although it completely blocked presentation to clone ALT81, indicating that the former epitope (TT947-967) is loaded on preformed and recycling molecules, whereas the latter (TT924-938) is loaded on newly synthesized molecules. When Epstein-Barr virus (EBV)-B cells were used as APC, 20-fold higher concentrations of TT and longer incubation times ( 1 and $4 \mathrm{~h}$ for clones ALT220 and ALT81, respectively) were required (data not shown). These results indicate that immature dendritic cells can use both recycling as well as newly synthesized class II molecules for rapid and efficient presentation of incoming antigen.

Immature dendritic cells synthesize class II molecules at a high rate (3-5 times faster than EBV-B cells; data not shown) and this rate is further increased by maturation stimuli. As shown in Fig. 4a, a 2-3-fold increase in class II synthesis was observed as early as one hour after challenge with LPS or TNF- $\alpha$ and was sustained for 1016 hours. But at later times, class II synthesis was completely shut off. MHC class I synthesis was also upregulated after maturation
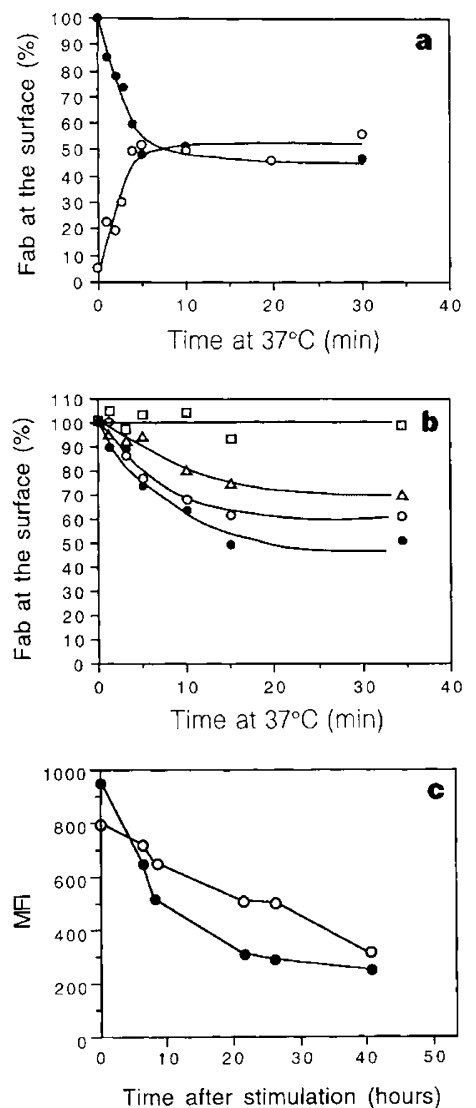

Figure 2 Extensive internalization and recycling of MHC class II molecules in immature DC is progressively lost together with endocytic activity following maturation. a, Internalization of surface-bound $(\bullet)$ and recycling of internalized (O) L243 biotinylated Fab fragments in immature DC. Recycling is calculated as per cent of internalized Fab ${ }^{10}$. Comparable results were obtained in three different experiments. b. Progressive loss of class II internalization in DC stimulated for 0 $(\bullet), 4(O), 16(\triangle)$ and $40 \mathrm{~h}(\square)$ with LPS. Comparable results were obtained using TNF- $\alpha$. c, Accumulation of FITC-dextran (O) or Lucifer yellow $(\bullet)$ in DC stimulated with TNF- $\alpha$. Comparable results were obtained using LPS. MFI, mean fluorescence intensity. 
and was sustained for up to 64 hours (Fig. 4b). Total protein synthesis showed only small fluctuations (data not shown).

Our results indicate that in the first few hours after induction of maturation, dendritic cells are still able to internalize antigen at a high rate and have a high capacity for peptide loading as they still retain a large amount of recycling class II molecules while building up an increased pool of newly synthesized class II molecules. We therefore investigated whether inflammatory stimuli given together with the antigen could boost the formation of complexes between antigenic peptides and class II molecules. Immature dendritic cells were exposed to ${ }^{125} \mathrm{I}$-labelled TT for 10 hours in the presence or absence of the maturation stimulus. Addition of LPS gave a 3-7fold increase in the amount of SDS-stable class II dimers containing iodinated peptides (Fig. 5a, d). During the same period, total SDSstable dimers only increased $<2$-fold (Fig. $5 b-d$ ). These results show that the maturation stimulus enhances the generation of antigenic complexes.

In the APC analysed so far, class II molecules and most peptideclass II complexes are very stable and turn over with the same halflife of $\sim 30-40$ hours $^{16,17}$. As this stability is critical to immunogenicity, we tested whether the maturation process could also affect class II half-life. Immature dendritic cells were pulse-labelled with ${ }^{35} \mathrm{~S}$-methionine/cysteine and chased in the presence or absence of LPS. As shown in Fig. 6a, b and e, newly synthesized class II molecules disappear rapidly in immature dendritic cells, with a half-life of $\sim 10$ hours (range, 8 to 18 hours in 5 experiments). Addition of LPS during the chase period increased class II half-life to more than 100 hours. In contrast, the half-life of class I molecules was not affected by maturation (Fig. 6f). Likewise, after cell-surface iodination, there was a comparable shift in the half-life of class II

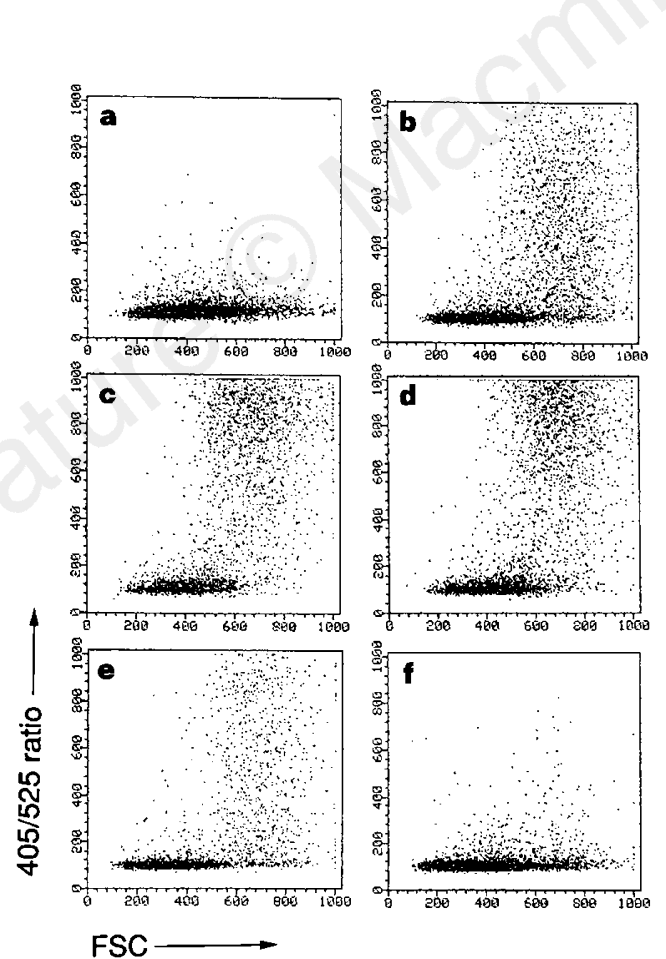

Figure $3 \mathrm{Immature} \mathrm{DC}$ can efficiently load antigenic peptides on both recycling and newly synthesized MHC class II molecules. Immature DC were pulsed with $2 \mu \mathrm{g} \mathrm{ml}^{-1}$ TT and tested at different times for their ability to trigger an increase in $\left[\mathrm{Ca}^{2+}\right]_{i}$ in T-cell clones ALT220, specific for TT947-967 (a-d) and ALT81, specific for TT924-938 (e, f). DC were either unpulsed (a) or pulsed with TT for $20 \mathrm{~min}$ (b), $40 \mathrm{~min}(\mathbf{c}, \mathbf{d})$ or $60 \mathrm{~min}(\mathbf{e}, \mathbf{f})$. In $\mathbf{d}$ and $\mathbf{f}$, DC were pretreated for $7 \mathrm{~h}$ with $10 \mu \mathrm{g} \mathrm{ml}^{-1}$ cycloheximide. The epitope recognized by clone ALT220 was generated in 15$20 \mathrm{~min}$, whereas that recognized by clone ALT81 appeared after 40-50 min. When EBV-B cells were used as APC, the dose of antigen required was at least 20 -fold higher and generation of the epitopes was delayed. molecules (Fig. 6c, d), indicating that the short half-life observed in immature dendritic cells is mainly due to degradation of class II molecules that had already reached the cell surface. We also measured the efficiency of transport in immature dendritic cells of newly synthesized class II molecules to the cell surface by combining metabolic labelling with surface biotinylation ${ }^{18}$. As shown in Fig. 6g, newly synthesized class II molecules are efficiently transported to the cell surface. By correcting for the amount of material loaded, the efficiency of biotinylation and the presence of the recycling pool, we estimate that $>80 \%$ of newly synthesized class II molecules is delivered to the cell surface within 3 hours. Taken together with the functional data (Fig. 3), our results show that in immature dendritic cells newly synthesized class II molecules reach the cell surface loaded with antigenic peptides, but are subsequently degraded. Maturation results in a dramatic shift in class II half-life allowing accumulation and persistence of the complexes formed.

The stabilization of antigenic complexes following maturation was tested in a functional assay. As shown in Fig. 7, immature

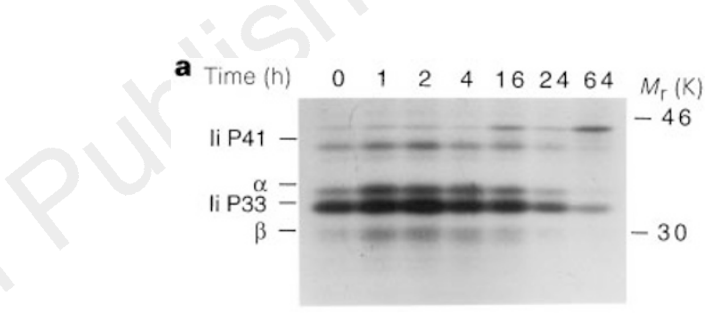

b

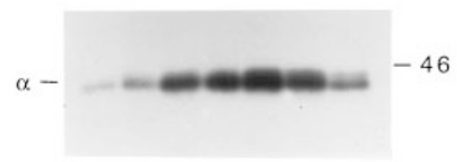

Figure 4 Transient upregulation of MHC class II synthesis in maturing DC. DC were metabolically labelled for $20 \mathrm{~min}$ before $(t=0)$ and at different times after LPS stimulation. a, MHC class II, and b, MHC class I immunoprecipitates separated by SDS-PAGE. The rate of increase in class II synthesis was 2-3fold and comparable for six DC preparations stimulated by either LPS or TNF- $\alpha$. li, invariant chain.
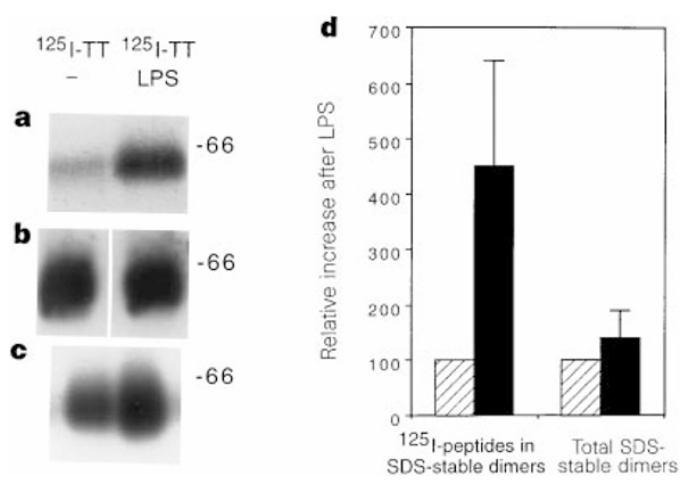

Figure 5 Maturation increases generation of SDS-stable class II dimers containing antigenic peptides. DC were incubated for $10 \mathrm{~h}$ with ${ }^{125}$-labelled TT or unlabelled TT in the presence or absence of LPS. a, SDS-stable dimers containing iodinated peptides; $\mathbf{b}$, total SDS-stable dimers measured in the same experiment by western blot developed with ${ }^{125}$ l-labelled protein A; c, total SDS-stable dimers in immature and LPS-treated (10 h) DC detected by cellsurface iodination; $\mathbf{d}$, quantification by Phosphorimager of ${ }^{125}$-peptides in SDSstable dimers (6 experiments), and of total SDS stable dimers (3 experiments) in untreated DC (hatched bars) and LPS-treated DC (black bars). 
dendritic cells can efficiently stimulate $T$ cells if tested immediately after pulsing with antigen, but lose this capacity over time. However, when LPS was added during the chase period, the same cells could still strongly stimulate T cells after 72 hours. Together these results indicate that antigenic memory is induced by the maturation process.

We have shown that different mechanisms coordinate to maximize the antigen-presenting capacity of dendritic cells over a short period following exposure to inflammatory stimuli. In the first 24 hours after maturation is induced, antigenic peptides are efficiently loaded onto the rapidly recycling class II pool and onto newly synthesized class II molecules, which are produced in increased amounts. There is a key shift in class II half-life, which, together with the increased rate of synthesis, results in the rapid accumulation of a large number of stable class II complexes that can be displayed for long periods, enabling dendritic cells to maintain long-term memory of past encounters with antigen.

The degradation of class II molecules in immature dendritic cells and their stabilization after maturation occur in both human and mouse. In murine 'early' dendritic cells, newly synthesized class II molecules are transported inefficiently to the cell surface and degraded, whereas in human immature dendritic cells the transport to the cell surface is relatively efficient and molecules that have already reached the plasma membrane are rapidly degraded. One explanation may be that degradation of class II molecules in human dendritic cells, is linked to their high rate of endocytosis ${ }^{8}$. A half-life of $\sim 10$ hours could be explained by the failure of as few as $0.5 \%$ of internalized class II molecules to recycle. In mature dendritic cells,

\section{a}

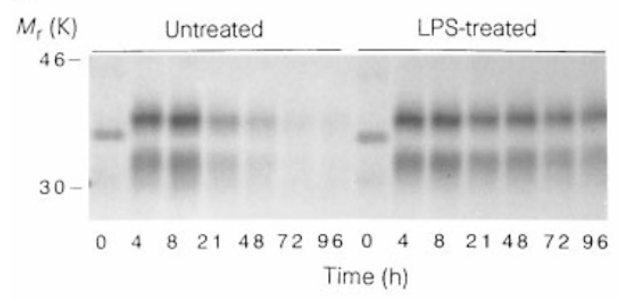

c

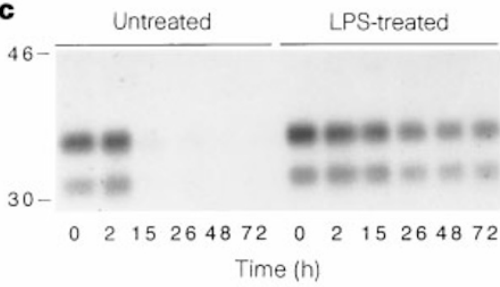

b

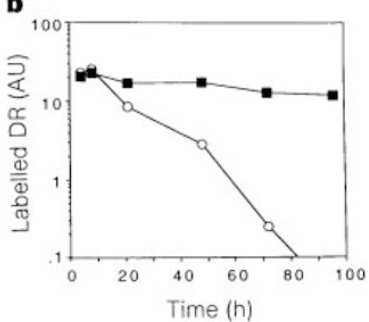

d

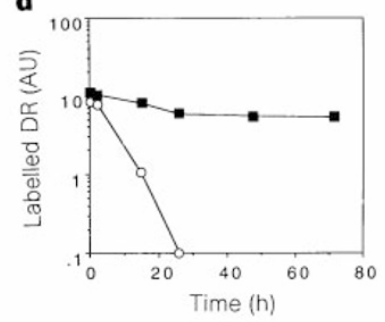

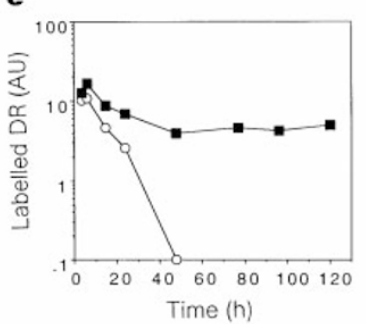

$\mathbf{f}$

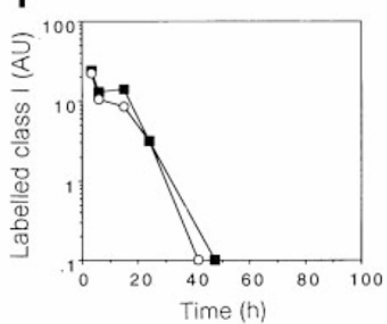

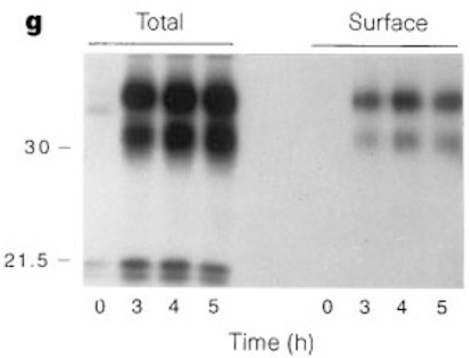

Figure 6 Maturation stimuli increase the half-life of class II molecules from $\sim 10$ to $>100$ hours. a, Stability of biosynthetically labelled class II molecules in the absence or in the presence of LPS, and $\mathbf{b}$, quantification by Phosphorimager of the radioactivity associated with the $\alpha$-chains in immature DC (O) or in DC chased in the presence of LPS (घ) AU, arbitrary units. c, Stability of surface-iodinated class II molecules in the absence or in the presence of LPS, and $\mathbf{d}$, quantification. e, $\mathbf{f}$, Stability of biosynthetically labelled class II molecules (e) and class I molecules (f) measured in the same experiment. $\mathbf{g}$, Efficient transport of newly synthesized class II molecules to the cell surface in immature DC. DC were biosynthetically labelled and surface-biotinylated after $0,3,4$ and 5 hours of chase. The class II immunoprecipitates were analysed as such (left) or after reprecipitation with streptavidin-Sepharose (right). The amount of class II molecules delivered to the cell surface (>80\% in $3 \mathrm{~h}$ ) was calculated as described ${ }^{18}$. 

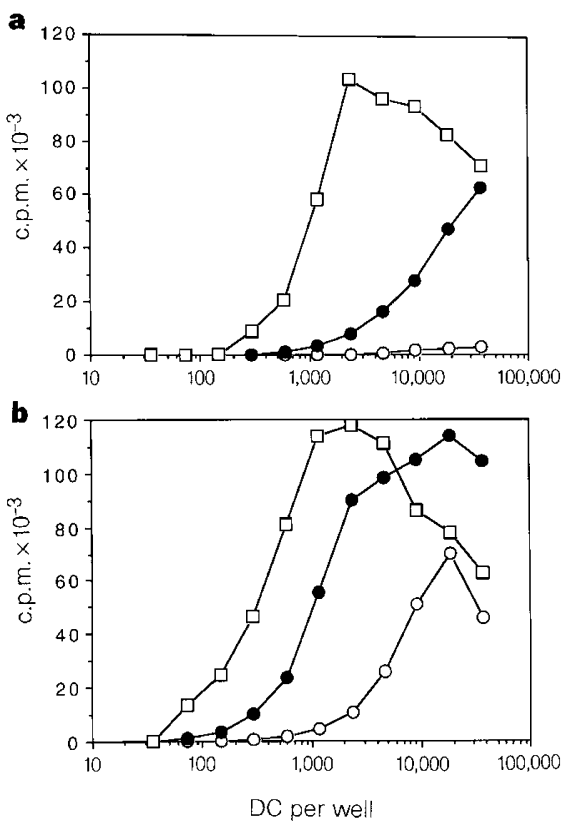

Figure 7 Maturation allows DC to maintain memory of antigens encountered. Immature DC were pulsed with $\mathbf{a}, 5 \mu \mathrm{g} \mathrm{ml}^{-1} \mathrm{TT}$, or $\mathbf{b}, 0.1 \mu \mathrm{M}$ TT830-843 peptide for $3 \mathrm{~h}$ at $37^{\circ} \mathrm{C}$, washed and either tested immediately ( $\left.\square\right)$, or re-cultured in the presence $(\bullet)$ or absence $(O)$ of LPS for $72 \mathrm{~h}$ before being tested for their ability to stimulate a specific T-cell clone KS140. Note that the clone does not express $\mathrm{CD} 28$ and is thus insensitive to co-stimulation.

decreased endocytosis may account for the very long half-life (over 100 hours) which exceeds that estimated in other cell types ${ }^{16,17}$. Class II internalization and recycling may thus have the dual function of allowing rapid loading of peptides and of shortening the half-life of preformed molecules, so preventing the accumulation of complexes before encounter with the inflammatory stimulus. The shift in halflife of class II molecules in human and murine dendritic cells ensures that only those epitopes generated at sites of inflammation will be displayed in lymphoid organs for T-cell priming.

\section{Methods}

Dendritic cells. Immature dendritic cells (DC) were prepared as described ${ }^{7,8}$. Peripheral blood monocytes purified by centrifugal elutriation were cultured in RPMI- 1640 supplemented with $10 \% \mathrm{FCS}, 50 \mathrm{ng} \mathrm{ml}^{-1} \mathrm{GM}-\mathrm{CSF}$ and $1,000 \mathrm{U} \mathrm{ml}^{-}$ ${ }^{1}$ IL-4 for 6-8 days. Maturation was induced by addition of either $1 \mu \mathrm{g} \mathrm{ml}^{-1} \mathrm{LPS}$ (from S. abortus equi, Sebak) or $50 \mathrm{ng} \mathrm{ml}^{-1}$ recombinant TNF- $\alpha$ (R\&D Systems). Uptake and accumulation of FITC-dextran or Lucifer yellow (Molecular Probes) have been described ${ }^{8}$.

Immunofluorescence. Cells were fixed for $30 \mathrm{~min}$ with $2 \%$ paraformaldehyde and then either permeabilized for $30 \mathrm{~min}$ with PBS containing $0.5 \%$ saponin, $5 \%$ FCS and $10 \mathrm{mM}$ HEPES, or stained. Cells were stained with saturating concentrations of L243 anti-DR antibody (ATCC), followed by FITC-labelled goat anti-mouse IgG2a (Southern Biotechnology Associates). The number of DR molecules was calculated by extrapolation of the calibration curve obtained using beads coated with known amounts of mouse IgG2a (Qifikit, DAKO).

Internalization and recycling of class II molecules. Fab fragments of L243 antibody were biotinylated with NHS-SS-biotin (Pierce) $)^{10}$. DC were incubated at $0^{\circ} \mathrm{C}$ for $2 \mathrm{~h}$ with $30 \mu \mathrm{g} \mathrm{ml}^{-1} \mathrm{Fab}$, washed in cold medium, shifted at $37^{\circ} \mathrm{C}$ for various times and rapidly transferred to cold medium. The amount of Fab remaining on the cell surface was determined using ${ }^{125}$ I-labelled streptavidin (Amersham) as described ${ }^{10}$. To detect recycling, bound Fab were allowed to equilibrate for $20 \mathrm{~min}$ at $37^{\circ} \mathrm{C}$. Cells were chilled and surface biotin was removed using two rounds of treatment with $50 \mathrm{mM}$ glutathione (Sigma). The cells were then shifted to $37^{\circ} \mathrm{C}$ for various times and the biotinylated Fab reappearing on the cell surface detected as described.

Class II synthetic rate. At different times after stimulation with TNF- $\alpha$ or LPS, DC were labelled with ${ }^{35} \mathrm{~S}$-methionine/cysteine (Amersham) for $20 \mathrm{~min}$.
Cells were lysed and MHC class II molecules immunoprecipitated using a polyclonal anti-class II rabbit antiserum (from $\mathrm{H}$. Ploegh) and protein ASepharose.

Loading of antigenic peptides on SDS-stable class II dimers. TT (Connaugh) was iodinated to a specific activity of $5 \times 10^{4}$ c.p.m. per ng with ${ }^{125} \mathrm{I}$ using the iodogen method (Pierce) $)^{9} .3 \times 10^{6}$ DC were incubated with ${ }^{125} \mathrm{I}-$ TT ( $3 \times 10^{8}$ c.p.m. $)$ in the absence or presence of LPS. After $10 \mathrm{~h}$, the cells were washed and lysed and class II molecules were precipitated with DA6.231 (antiDR) antibody. Class II molecules were eluted at $24^{\circ} \mathrm{C}$ with $2 \%$ SDS buffer under non-reducing conditions and analysed by SDS-PAGE ${ }^{19}$. Replica gels were transferred to nitrocellulose and total levels of class II molecules were measured using a rabbit anti-class II antiserum followed by ${ }^{125}$ I-protein A (Amersham) and quantified by using a Phosphorimager.

Half-life of class II molecules. Immature DC were either biosynthetically labelled with ${ }^{35} \mathrm{~S}$-methionine/cysteine for $20 \mathrm{~min}$ or surface-iodinated by the SHPP method ${ }^{13}$ and cultured in the presence or absence of LPS. After various times of chase, the same number of cells were lysed and MHC class II molecules were precipitated with L243 mAb and analysed by SDS-PAGE under reducing conditions. The radioactivity in specific bands was quantified by Phosphorimager. DC do not divide during the assay.

Transport of newly synthesized class II molecules to the cell surface. This assay was done as described ${ }^{18}$. Immature DC were labelled with ${ }^{35} \mathrm{~S}$-methionine/cysteine for $20 \mathrm{~min}$ and chased in normal medium. At 0, 3, 4 and $5 \mathrm{~h}$, cells were surface-biotinylated using $2 \mathrm{mg} \mathrm{ml}^{-1}$ NHS-SS-biotin (Pierce) on ice for $11 \mathrm{~min}$. After washing, the cells were lysed and class II molecules were immunoprecipitated with L243 mAb. Precipitates were boiled in $100 \mu$ PBS $2 \%$ SDS. $30 \mu \mathrm{l}$ were analysed directly (total class II) and $70 \mu \mathrm{l}$ were diluted into $1 \mathrm{ml}$ PBS 2\% Triton X100 and reprecipitated with streptavidin-Sepharose (Zymed) (surface biotinylated class II). The efficiency of transport to the cell surface was calculated ${ }^{18}$ by considering the amount of material loaded, the efficiency of biotinylation $(\sim 30 \%)$ and the presence of an internalized pool of class II molecules.

Antigen-presentation assay. To measure the kinetics of antigen processing ${ }^{15}$, DC were incubated with $2 \mu \mathrm{g} \mathrm{ml}^{-1} \mathrm{TT}$ at $37^{\circ} \mathrm{C}$ and tested at different times for their ability to trigger an increase in $\left[\mathrm{Ca}^{2+}\right]$ in TT-specific T-cell clones loaded with Indol AM (Sigma). Cycloheximide $\left(10 \mu \mathrm{g} \mathrm{ml}^{-1}\right.$; Sigma) was added to DC 7 hours before the assay. To estimate the persistence of specific peptide-MHC complexes, DC were pulsed with TT protein or TT830-843 peptide and cultured in the presence or absence of LPS for $72 \mathrm{~h}$. Graded numbers of cells were tested for their capacity to trigger T-cell proliferation of a specific T-cell clone. Clones used in combination with autologous or class II-matched DC were ALT220 (specific for TT947-967), ALT81 (TT924-938) and KS140 (TT830-843).

Received 19 March; accepted 28 May 1997

1. Steinman, R. M. The dendritic cell system and its role in immunogenicity. Annu. Rev. Immunol. 9, 271-296 (1991).

2. Pure, E. et al. Antigen processing by epidermal Langerhans cells correlates with the level of biosynthesis of major histocompatibility complex class II molecules and expression of invariant chain. J. Exp. Med. 172, 1459-1469 (1990).

3. Roake, J. A. et al. Dendritic cell loss from nonlymphoid tissues after systemic administration of lipopolysaccharide, tumor necrosis factor, and interleukin 1. J. Exp. Med. 181, 2237-2247 (1995).

4. Stossel, H. et al. Disappearance of certain acidic organelles (endosomes and Langerhans cell granules) accompanies loss of antigen processing capacity upon culture of epidermal Langerhans cells. J. Exp. Med. 172, 1471-1482 (1990).

5. Kampgen, E. et al. Class II major histocompatibility complex molecules of murine dendritic cells: synthesis, sialylation of invariant chain, and antigen processing capacity are down-regulated upon culture. Proc. Natl Acad. Sci. USA 88, 3014-3018 (1991).

6. Enk, A. H. \& Katz, S. I. Early molecular events in the induction phase of contact sensitivity. Proc. Natl Acad. Sci. USA 89, 1398-1402 (1992).

7. Sallusto, F. \& Lanzavecchia, A. Efficient presentation of soluble antigen by cultured human dendritic cells is maintained by granulocyte/macrophage colony-stimulating factor plus interleukin 4 and downregulated by tumor necrosis factor alpha. J. Exp. Med. 179, 1109-1118 (1994).

8. Sallusto, F., Cella, M., Danieli, C. \& Lanzavecchia, A. Dendritic cells use macropinocytosis and the mannose receptor to concentrate macromolecules in the major histocompatibility complex class II compartment: downregulation by cytokines and bacterial products. J. Exp. Med. 182, 389-400 (1995).

9. Davidson, H. W., Reid, P. A., Lanzavecchia, A. \& Watts, C. Processed antigen binds to newly synthesized MHC class II molecules in antigen-specific B lymphocytes. Cell 67, 105-116 (1991).

10. Pinet, V., Vergelli, M., Martin, R., Bakke, O. \& Long, E. O. Antigen presentation mediated by recycling of surface HLA-DR molecules. Nature 375, 603-606 (1995).

11. Pinet, V., Malnati, M. S. \& Long, E. O. Two processing pathways for the MHC class II-restricted presentation of exogenous influenza virus antigen. J. Immunol. 152, 4852-4860 (1994).

12. Zhong, G., Romagnoli, P. \& Germain, R. N. Related leucine-based cytoplasmic targeting signals in Invariant chain and major histocompatibiity complex class II molecules control endocytic presentation of distinct determinants in a single protein. J. Exp. Med. 185, 429-438 (1997). 
3. Reid, P. A. \& Watts, C. Cycling of cell-surface MHC glycoproteins through primaquine-sensitive intracellular compartments. Nature 346, 655-657 (1990)

14. Neefjes, J. J., Stollorz, V., Peters, P. J., Geuze, H. J. \& Ploegh, H. L. The biosynthetic pathway of MHC class II but not class I molecules intersects the endocytic route. Cell 61, 171-183 (1990).

15. Roosnek, E., Demotz, S., Corradin, G. \& Lanzavecchia, A. Kinetics of MHC-antigen complex formation on antigen-presenting cells. Immunol. 140, 4079-4082 (1988).

16. Lanzavecchia, A., Reid, P. A. \& Watts, C. Irreversible association of peptides with class II MHC molecules in living cells. Nature 357, 249-252 (1992).

17. Nelson, C. A., Petzold, S. J. \& Unanue, E. R. Peptides determine the lifespan of MHC class II molecules in the antigen-presenting cell. Nature 371, 250-252 (1994).

18. Amigorena, S., Drake, J. R., Webster, P. \& Mellman, I. Transient accumulation of new class II MHC molecules in a novel endocytic compartment in B lymphocytes. Nature 369, 113-120 (1994).

19. Germain, R. N. \& Hendrix, L. R. MHC class II structure, occupancy and surface expression determined by post-endoplasmic reticulum antigen binding. Nature 353, 134-139 (1991).

20. Pierre, P. et al. Developmental regulation of MHC class II transport in mouse dendritic cells. Nature this issue. EDS to complete on page

Acknowledgements. We thank D. Scheidegger and M. Dessing for technical assistance, E. Long for discussion, and C. Watts, F. Sallusto, K. Karjalainen and M. Colonna for critically reading the manuscript. The Basel Institute for Immunology was founded and is supported by F. Hoffmann-La Roche, Basel, Switzerland.

Correspondence and requests for material should be addressed to M.C. (e-mail: cella@bii.ch).

\section{Developmental regulation} of MHC class II transport in mouse dendritic cells

\section{Philippe Pierre*, Shannon J. Turley ${ }^{\star}$, Evelina Gatti, Michael Hull, Joseph Meltzer $\dagger$, Asra Mirza $\dagger$, Kayo Inaba $\dagger$, Ralph M. Steinman $\dagger$ \& Ira Mellman}

Department of Cell Biology, Yale University School of Medicine, 333 Cedar Street, PO Box 208002, New Haven, Connecticut 06520, USA

$\dagger$ The Rockefeller University, 1230 York Avenue, New York, New York 10021, USA * These authors contributed equally to this work.

Dendritic cells (DCs) have the unique capacity to initiate primary and secondary immune responses ${ }^{1-3}$. They acquire antigens in peripheral tissues and migrate to lymphoid organs where they present processed peptides to $T$ cells. DCs must therefore exist in distinct functional states, an idea that is supported by observations that they downregulate endocytosis and upregulate surface molecules of the class II major histocompatibility complex (MHC) upon maturation ${ }^{4-7}$. Here we investigate the features of DC maturation by reconstituting the terminal differentiation of mouse DCs in vitro and in situ. We find that early DCs, corresponding to those found in peripheral tissues, exhibit a phenotype in which most class II molecules are intracellular and localized to lysosomes. Upon maturation, these cells give rise to a new intermediate phenotype in which intracellular class II molecules are found in peripheral non-lysosomal vesicles, similar to the specialized CIIV population seen in B cells. The intermediate cells then differentiate into late DCs which express almost all of their class II molecules on the plasma membrane. These variations in class II compartmentalization are accompanied by dramatic alterations in the intracellular transport of the new class II molecules and in antigen presentation. We found that although early DCs could not present antigen immediately after uptake, efficient presentation of the previously internalized antigen occurred after maturation, 24-48 hours later. By regulating class II transport and compartmentalization, DCs are able to delay antigen display, a property crucial to their role in immune surveillance.

Mouse bone marrow is a major source of DCs when cultivated with granulocyte-macrophage colony-stimulating factor (GM$\mathrm{CSF})^{8}$. Immunofluorescence microscopy of these cultures revealed three distinct developmental stages. Cells were identified as DCs by the expected repertoire of antigens and expression of MHC class II, cell shape, and adherence. As reported previously ${ }^{8}$, DCs were found by immunofluorescence or FACS to be negative or weakly positive for the granulocyte marker GR1, negative for the macrophage marker SER-4, but strongly positive for CD11c and MHC class II. Contaminating SER-4 or GR1-positive cells were negative for class II and judged not to be DCs.

After 4-5 days, DCs were found in proliferating clusters loosely attached to adherent stromal cells ${ }^{8}$. By confocal microscopy, most of the cells present in or migrating out from the clusters showed little MHC class II on their surface, but contained abundant intracellular class II (Fig. 1a). The class II-positive vesicles represented lysosomes (MIICs) and late endosomes, being positive for lgp-B/lamp-2 and $\mathrm{H} 2-\mathrm{M}$ (Fig. 1a). Thus, they were characteristic of MIIC as defined in human lymphoblasts and human $\mathrm{DCs}^{9-12}$. As the MIIC-containing cells were present in proliferating clusters, we defined them as 'early' DCs.

With increasing time in culture, two additional cell populations were detected. The first of these ('intermediate' DCs) was present transiently and comprised non-adherent cells that had little surface MHC class II (Fig. 1b). They were strikingly unlike the early cells, however, because most of their intracellular class II was in a vesicle population that was devoid of lysosomal markers (Fig. 1b, arrows), and thus reminiscent of non-lysosomal, class II-positive CIIV isolated from A20 B cells ${ }^{13}$. At later times in particular, the vesicles accumulated directly beneath the plasma membrane (Fig. 1b, right), whereas the lysosomes became concentrated in the perinuclear region.

The third major DC population accumulated with time until by 8-10 days it represented almost all of the non-adherent class IIpositive cells. These 'late' cells had a more classical DC phenotype, with long processes that stained for class II (green) (Fig. 1c). Little class II remained intracellularly, with most of the now largely class II-depleted H2-M/lamp-positive lysosomes visualized as poorly resolved clusters of red-staining vesicles in the perinuclear region. Further characterization indicated that markers such as DEC-205 and $2 \mathrm{~A} 1$ were absent from early cells but expressed at moderate and high levels on intermediate and late cells, respectively ${ }^{8,14}$ (results not shown). Early cells, but not late cells, were capable of efficient fluid endocytosis (W. Garrett and I.M., unpublished results), as found previously for human cells ${ }^{4}$.

To determine whether early cells pass through the intermediate phenotype before reaching maturity, we produced highly purified populations of early cells by gently dislodging and isolating proliferating clusters on serum columns, followed by depletion of contaminating cells by fluorescent-activated cell sorting (FACS) ${ }^{8}$. This approach yielded $\sim 95 \%$ pure populations of early DCs. As quantified in Fig. 1d, after 5-8 h in culture, the early cells had nearly disappeared and $>60 \%$ of the population of exhibited the intermediate phenotype (for example, see Fig. 1b); 20-30\% exhibited the late or mature phenotype (as shown in Fig. 1c). After $24 \mathrm{~h}$, $\sim 90 \%$ of the cells were found to be of the late phenotype. As the number of cells remained constant throughout, these results strongly suggest that there is a sequential relationship in the maturation pathway. The rapid maturation kinetics observed using purified cluster-derived cells probably reflect their greater developmental synchrony.

To ensure that the developmental sequence was not peculiar to bone marrow cultures, we investigated whether tissue DCs had similar properties. We first examined epidermal Langerhans cells ${ }^{15}$. In epidermal explants, class II was present in these cells in a punctuate pattern which co-localized with lysosomal marker H2$\mathrm{M}$, reminiscent of early bone marrow DCs (Fig. 2, upper right panels). If explants were incubated in culture medium and the Langerhans cells allowed to mature in situ, within $4 \mathrm{~h}$ the degree of class II and H2-M co-localization decreased, with class II staining remaining punctuate but becoming progressively less coincident with $\mathrm{H} 2-\mathrm{M}$, which became concentrated at the cell body (Fig. 2, right panels). This pattern was consistent with the intermediate 